\title{
RADIATION IN BYTHINELLA MOQUIN-TANDON, 1856 (MOLLUSCA: GASTROPODA: RISSOOIDEA) IN THE BALKANS
}

\author{
ANDRZEJ FALNIOWSKI ${ }^{1 *}$, MAGDAlENA SZAROWSKA ${ }^{1}$, PETER GLÖER ${ }^{2}$, Vladimir PEŠIĆ ${ }^{3}$, \\ DILIAN GEORGIEV ${ }^{4}$, MICHAL HORSÁK ${ }^{5}$, IOAN SIRBU ${ }^{6}$
}

\author{
${ }^{1}$ Department of Malacology, Institute of Zoology, Jagiellonian University, Gronostajowa 9, 30-387 Kraków, \\ Poland (e-mail: andrzej.falniowski@uj.edu.pl) \\ ${ }^{2}$ Schulstr. 3, D-25491 Hetlingen, Germany \\ ${ }^{3}$ Department of Biology, University of Montenegro, Cetinjski put b.b, 81000 Podgorica, Montenegro \\ ${ }^{4}$ Department of Ecology and Environmental Conservation, University of Plovdiv, Tzar Assen St. 24, \\ BG-4000 Plovdiv, Bulgaria \\ ${ }^{5}$ Department of Botany and Zoology, Masaryk University, Kotlářská 2, 61137 Brno, Czech Republic \\ ${ }^{6}$ Department of Ecology and Environmental Protection, Lucian Blaga University, 31 Oituz St., 550337 \\ Sibiu, Romania \\ *corresponding author
}

ABSTRACT: 61 sequences of cytochrome oxidase subunit 1 (COI), 570 bp long, from Central Europe (8), Slovenia (2), and from the Balkans (51: 6 from, Romania, 15 from Greece, 15 from Bulgaria, 6 from Serbia, and 9 from Montenegro), 33 of them new, together with 61 sequences of the ribosomal 18S, $450 \mathrm{bp}$ long, all of them new, were analysed to infer the pattern of radiation of Bythinella in the Balkans. Thirty two nominal taxa of Bythinella (22 nominal species: B. austriaca, B. calimanica, B. charpentieri, B. compressa, B. dacica, B. dispersa, B. grossui, B. hansboetersi, B. luteola, B. micherdzinskii, B. molcsanyi, B. nonveilleri, B. pannonica, B. pesterica, B. radomani, B. rhodopensis, B. robiciana, B. schmidti, B. slaveyae, B. srednogorica, B. taraensis and B. viseuiana; one nominal subspecies: B. austriaca ehrmanni; and nine Greek species not yet described) were included, represented mostly by paratypes or at least topotypes, collected at 31 Balkan localities. The phylogeny, inferred on the combined data set with the ML approach, showed two large clades, although they were weakly supported. One of them comprised the Romanian and Montenegro populations, and one Serbian population, the other (less genetically diversified) consisted of one Serbian and all the Bulgarian/Greek populations. The origin of those two clades was dated, with the external data, to be more than $4.341 \pm 0753$ MYA old, thus its origin was assigned to isolation by the Dacic Basin (part of Paratethys). All the Bulgarian populations presumably belong to one species, which may be assigned to the recent recolonisation of this territory from the south.

KEY WORDS: Rissooidea, Balkan Peninsula, DNA, phylogeny, taxonomy, speciation

\section{INTRODUCTION}

The genus Bythinella Moquin-Tandon, 1856 is distributed from west Europe (the Iberian Peninsula), to west Asia. These dioecious, oviparous snails with minute, dextral, ovoid shells inhabit freshwater springs, small brooks and groundwaters (GIUSTI \& PEZZOLI 1977, FALNIOWSKI 1987). They are found in abundance on mosses and other aquatic plants, fallen leaves, dead wood, etc. The rich literature on
Bythinella (RADOMAN 1976, 1983, 1985, GIUSTI \& PEZZOLI 1977, FALNIOWSKI 1987, 1992, GLÖER 2002, SZAROWSKA \& WILKE 2004) pertains mainly to western, southern and central Europe. The characters of either the shell or the soft parts do not allow for clear delineation of Bythinella species. Recently, BICHAIN et al. (2007a, b), HAASE et al. (2007), FALNIOWSKI et al. (2009b, c) and BENKE et al. (2009) applied molecular 
data and proved the distinctiveness of several species of the genus. The ranges of some Bythinella species studied molecularly so far (e.g. BICHAIN et al. 2007a, b, FALNIOWSKI et al. 2009b, c, BENKE et al. 2009, FALNIOWSKI \& SZAROWSKA 2011) are restricted to a single spring, spring complex, or local watershed, while those of the other congeners stretch across one or more drainage divides.

There were several species described and redescribed in central Europe (JUNGBLUTH \& BOETERS 1977, FALNiOWSKI 1987, 1992, GLÖER 2002, SZAROWSKA \& WILKE 2004, HAASE et al. 2007) and the former Yugoslavia (RADOMAN 1976, 1983, 1985, GLÖER 2008, GLÖER \& PEŠIĆ 2010). Recently we found six molecularly distinct species of this genus in Romania (FALNIOWSKI et al. 2009a, b) and ten molecularly distinct (9 of them not yet described) species in Greece (FALNIOWSKI \& SZAROWSKA 2011). Several nominal species were recently reported from Bulgaria (GLÖER \& PEŠIĆ 2006, FALNIOWSKI et al. 2009a, GLÖER \& GEORGIEV 2009) and Montenegro (GLÖER \& PEŠIĆ 2010).

FALNIOWSKI et al. (2009b, c) and FALNIOWSKI \& SZAROWSKA (2011) demonstrated that (1) the morphostatic mode of evolution as defined by DAVIS (1992) is common in Bythinella; (2) it is impossible to distinguish Bythinella species if there are no molecular data, although the morphology must be considered as

\section{MATERIALS AND METHODS}

\section{MATERIAL COLLECTION AND FIXATION}

We collected snails, with a sieve or by hand, at 30 localities in the Balkans (Fig. 1, Table 1). Snails were washed twice in $80 \%$ ethanol and left to stand in it for ca. 12 hours, after which the ethanol was changed twice in 24 hours and finally, after a few days, the $80 \%$ solution was replaced with a $96 \%$ one. The material was stored at $-20^{\circ} \mathrm{C}$.

\section{MOLECULAR TECHNIQUES APPLIED}

Snails were hydrated in TE buffer $(3 \times 10 \mathrm{~min}$. $)$ and their DNA was extracted with the SHERLOCK extracting kit (A\&A Biotechnology); the final product was dissolved in $20 \mu \mathrm{l}$ of TE buffer. The PCR reaction was performed with the following primers: LCOI490 (5'-GGTCAACAAATCATAAAGATATTGG-3') and COR722b (5'-TAAACTTCAGGGTGACCAAAAAA TYA-3') for the COI gene (FOLMER et al. 1994) and SWAM18SF1 (5'-GAATGGCTCATTAAATCAGTCGA GGTTCGTTAGATGATCCAAATC-3'), SWAM18SR1 (5'-ATCCTCGTTAAAGGGTTTAAAGTGTACTCATT CCAATTACGGAGC-3') for the $18 \mathrm{~S}$ gene (PALUMBI 1996). The PCR conditions were as follows: well (BICHAIN et al. 2007b, HAASE et al. 2007). In this study we applied mitochondrial cytochrome oxidase subunit I (COI), and ribosomal $18 \mathrm{~S}$ to infer the general pattern of radiation in this genus in the Balkan Peninsula. Most of the specimens sequenced were paratypes or at least topotypes of the nominal taxa; considering all the controversies concerning the species distinctness within Bythinella (GIUSTI \& PEZzOLI 1977, FALNIOWSKI 1987, BICHAIN et al. 2007a, b, HAASE et al. 2007, FALNIOWSKI et al. 2009b, c, FALNIOWSKI \& SZAROWSKA 2011), we leave unresolved the taxonomic status of those nominal taxa.

The Balkans once harboured one of the main European glacial refugia (HEWITT 1996, 2000, VOGEL et al. 1999, Petit et al. 2003, SCHMitT 2007, FALNiOWSKI et al. 2009b). Hence, they provide an interesting area for a study of radiation. In Romania (FALNIOWSKI et al. 2009a, b) we found greater genetic differences between Bythinella species than in Greece (FALNIOWSKI \& SZAROWSKA 2011). However, the data on the genus inhabiting the zone between Romania and Bulgaria are scarce (FALNIOWSKI et al. 2009a). Thus the aim of the present paper was to answer the following questions: (1) Can the pattern of genetic differentiation, decreasing from north to south, be applied to all the Balkans? (2) Does the pattern of (presumably) vicariant speciation reflect any palaeogeographic conditions in the Balkans?

1. For CO1: 4 min. at $94^{\circ} \mathrm{C}$ followed by 35 cycles of 1 min. at $94^{\circ} \mathrm{C}, 1 \mathrm{~min}$. at $55^{\circ} \mathrm{C}, 2 \mathrm{~min}$. at $72^{\circ} \mathrm{C}$, after all cycles an additional elongation step of $4 \mathrm{~min}$. at $72^{\circ} \mathrm{C}$ was performed;

2. For $18 \mathrm{~S}: 4 \mathrm{~min}$. at $94^{\circ} \mathrm{C}$ followed by 40 cycles of 45 sec. at $94^{\circ} \mathrm{C}, 45 \mathrm{sec}$. at $51^{\circ} \mathrm{C}, 2 \mathrm{~min}$. at $72^{\circ} \mathrm{C}$, after all cycles an additional elongation step of $4 \mathrm{~min}$. at $72^{\circ} \mathrm{C}$ was performed.

The total volume of each $\mathrm{PCR}$ reaction mixture was $50 \mu \mathrm{l}$. To check the quality of the PCR products, $10 \mu$ of the PCR product was run on $1 \%$ agarose gel. The PCR product was purified using Clean-Up columns (A\&A Biotechnology). The purified PCR product was dye-terminator amplified in both directions (HILlis et al. 1996) using BigDye Terminator v3.1 (Applied Biosystems), following the manufacturer's protocol and with the primers described above. The sequencing reaction products were purified using ExTerminator Columns (A\&A Biotechnology), and the sequences were read using an ABI Prism sequencer.

\section{DATA ANALYSIS}

The COI sequences were aligned by eye using BioEdit 5.0.0 (HALL 1999), and edited with MACCLADE 


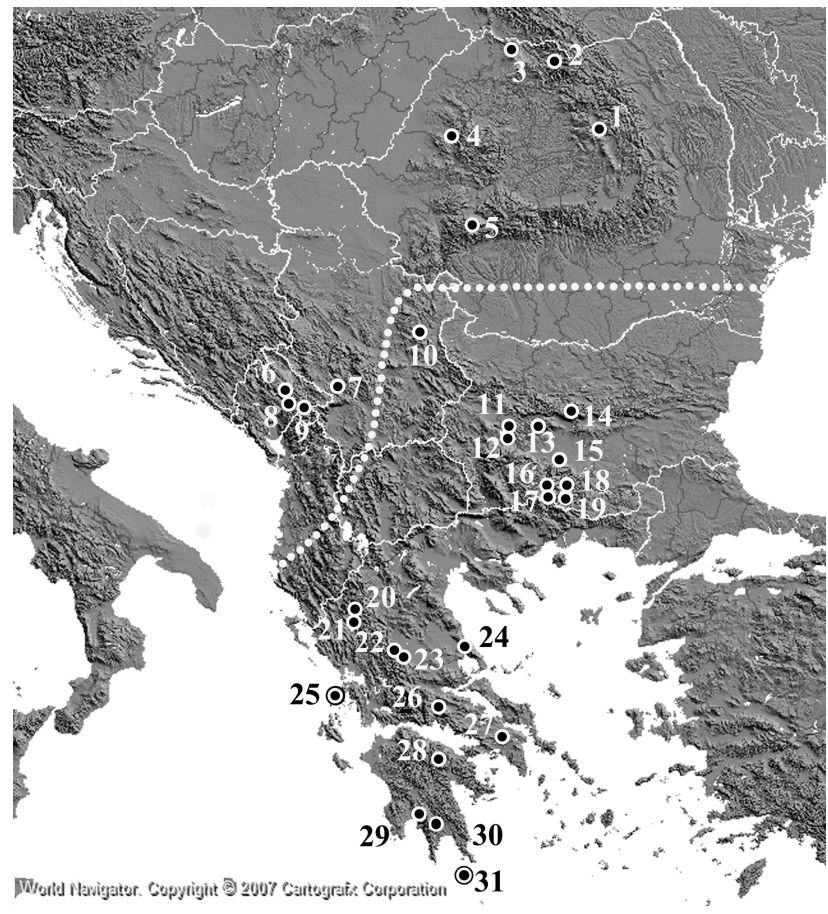

Fig. 1. Sampled localities of Bythinella in the Balkans. Localities numbers: see Table 1. Dotted line - border between two main clades (A and B). Figure produced using Cartografx Professional Software
4.05 (MADDISON \& MADDISON 2002). We examined mutational saturation for the COI dataset with saturation test of XIA et al. (XIA et al. 2003), performed with DAMBE 5.2.9 (XIA 2000). In 18 S alignment was performed using CLUSTALX 1.82 (THOMPSON et al. 1997).

Phylogenies of COI and 18S haplotypes were inferred using maximum-likelihood (ML), minimum evolution (ME) and maximum parsimony.

The maximum-likelihood technique of phylogeny reconstruction (SWOFFORD et al. 1996, NEI \& KUMAR 2000, FALNIOWSKI 2003) although criticised, is commonly used for molecular data and considered the most reliable one, so we decided to use the ML approach for each of the two data sets. For each maximum likelihood analysis, we tested different models of sequence evolution using Modeltest v3.06 (POSADA \& CRANDALl 1998, POSADA 2003). Following the recommendations of POSADA \& BUCKLEY (2004) and SOBER (2002), the best model for each dataset was chosen, using the Akaike Information Criterion (AKAIKE 1974). ML analyses were performed in PAUP*4.0b10 (SWOFFORD 2002), using an heuristic search strategy with stepwise addition of taxa, 10 random-sequence addition replicates, and tree-bisection-reconnection (TBR) branch swapping (SWOFFORD et al. 1996). Nodal support was esti-

Table 1. Localities and taxa studied; $\mathrm{N}$ - number of COI sequences used for phylogenetic inferrence; sequences not published earlier marked with aterisk

\begin{tabular}{|c|c|c|c|c|c|c|}
\hline No. & Locality description & $\begin{array}{l}\text { Geographical } \\
\text { coordinates }\end{array}$ & $\begin{array}{l}\text { Heigth } \\
\text { a.s.l. }\end{array}$ & Taxon & $\begin{array}{l}\text { Com- } \\
\text { ments }\end{array}$ & $\mathrm{N}$ \\
\hline 1 & $\begin{array}{l}\text { Călimani Mts, near Sălard village, } \\
\text { Toplita-Dead Gorges of the Mureş } \\
\text { River, Romania }\end{array}$ & $\begin{array}{l}46^{\circ} 57^{\prime} 10^{\prime \prime} \mathrm{N} \\
25^{\circ} 04^{\prime} 07^{\prime \prime} \mathrm{E}\end{array}$ & $620 \mathrm{~m}$ & $\begin{array}{l}\text { Bythinella calimanica } \\
\text { Falniowski, Szarowska et } \\
\text { Sirbu, } 2009\end{array}$ & paratype & 1 \\
\hline 2 & $\begin{array}{l}\text { Visseu River Valley, near Bistra, } \\
\text { Romania }\end{array}$ & $\begin{array}{l}47^{\circ} 52^{\prime} 14^{\prime \prime} \mathrm{N} \\
24^{\circ} 11^{\prime} 23 " \mathrm{E}\end{array}$ & $362 \mathrm{~m}$ & $\begin{array}{l}\text { Bythinella viseuiana Falniowski, } \\
\text { Szarowska et Sirbu, } 2009\end{array}$ & paratype & 1 \\
\hline 3 & $\begin{array}{l}\text { Izvoare Resort (Igniş Mountains), } \\
\text { Mara River Basin, Romania } \\
\text { (sympatrically) }\end{array}$ & $\begin{array}{l}47^{\circ} 44^{\prime} 51 " \mathrm{~N} \\
23^{\circ} 43^{\prime} 03^{\prime \prime} \mathrm{E}\end{array}$ & $909 \mathrm{~m}$ & $\begin{array}{l}\text { Bythinella grossui Falniowski, } \\
\text { Szarowska et Sirbu, } 2009 \\
\text { Bythinella molcsanyi H. Wagner, } \\
1941\end{array}$ & $\begin{array}{l}\text { paratype } \\
\text { topotype }\end{array}$ & 1 \\
\hline 4 & $\begin{array}{l}\text { Bihor Mountains, close to Vârtop } \\
\text { Pass, Crişul Băița River Basin, } \\
\text { Romania }\end{array}$ & $\begin{array}{l}46^{\circ} 31^{\prime} 25^{\prime \prime} \mathrm{N} \\
22^{\circ} 37^{\prime} 25^{\prime \prime} \mathrm{E}\end{array}$ & $1142 \mathrm{~m}$ & $\begin{array}{l}\text { Bythinella radomani Falniowski, } \\
\text { Szarowska et Sirbu, } 2009\end{array}$ & paratype & 1 \\
\hline 5 & $\begin{array}{l}\text { Retezat Mts Natural Park, La Beci, } \\
\text { Buta River Valley, Romania }\end{array}$ & $\begin{array}{l}45^{\circ} 18^{\prime} 26^{\prime \prime} \mathrm{N} \\
22^{\circ} 56^{\prime} 12^{\prime \prime} \mathrm{E}\end{array}$ & $1181 \mathrm{~m}$ & Bythinella dacica Grossu, 1946 & & 1 \\
\hline 6 & $\begin{array}{l}\text { Canyon of the River Tara, stream } \\
\text { Ljevok, Montenegro }\end{array}$ & $\begin{array}{l}42^{\circ} 59^{\prime} 29^{\prime \prime} \mathrm{N} \\
19^{\circ} 25^{\prime} 53^{\prime \prime} \mathrm{E}\end{array}$ & $835 \mathrm{~m}$ & $\begin{array}{l}\text { Bythinella taraensis Glöer et } \\
\text { Pešić, } 2010\end{array}$ & paratype & $3^{*}$ \\
\hline 7 & $\begin{array}{l}\text { Pester Plateau, Djerekare village, } \\
\text { Stračijevac Hill, Stračijevac spring, } \\
\text { Serbia }\end{array}$ & $\begin{array}{l}43^{\circ} 00^{\prime} \mathrm{N} \\
20^{\circ} 08^{\prime} \mathrm{E}\end{array}$ & $1350 \mathrm{~m}$ & Bythinella pesterica Glöer, 2008 & paratype & $3^{*}$ \\
\hline 8 & $\begin{array}{l}\text { National Park Biogradska Gora, } \\
\text { Bjelasica Mt., Montenegro }\end{array}$ & $\begin{array}{l}42^{\circ} 53^{\prime} 31^{\prime \prime} \mathrm{N} \\
19^{\circ} 36^{\prime} 16^{\prime \prime} \mathrm{E}\end{array}$ & $1119 \mathrm{~m}$ & $\begin{array}{l}\text { Bythinella luteola Radoman, } \\
1976\end{array}$ & & $3 *$ \\
\hline 9 & $\begin{array}{l}\text { Spring near Biogradsko Lake, } \\
\text { Montenegro Berane, spring in } \\
\text { Petnjik village, Montenegro }\end{array}$ & $\begin{array}{l}42^{\circ} 49^{\prime} 35^{\prime \prime} \mathrm{N} \\
19^{\circ} 54^{\prime} 10^{\prime \prime} \mathrm{E}\end{array}$ & $988 \mathrm{~m}$ & $\begin{array}{l}\text { Bythinella dispersa Radoman, } \\
1976\end{array}$ & & $3 *$ \\
\hline
\end{tabular}


Table 1. continued

\begin{tabular}{|c|c|c|c|c|c|c|}
\hline No. & Locality description & $\begin{array}{l}\text { Geographical } \\
\text { coordinates }\end{array}$ & $\begin{array}{l}\text { Heigth } \\
\text { a.s.l. }\end{array}$ & Taxon & $\begin{array}{l}\text { Com- } \\
\text { ments }\end{array}$ & $\mathrm{N}$ \\
\hline 10 & $\begin{array}{l}\text { Rtanj Mt, Vrmd a gorge, Vrmd a } \\
\text { spring, Serbia }\end{array}$ & $\begin{array}{l}43^{\circ} 42^{\prime} \mathrm{N} \\
21^{\circ} 49^{\prime} \mathrm{E}\end{array}$ & $600 \mathrm{~m}$ & $\begin{array}{l}\text { Bythinella nonveilleri Glöer, } \\
2008\end{array}$ & paratype & $3^{*}$ \\
\hline 11 & $\begin{array}{l}\text { Anton town, below Bolovan Hill, } \\
\text { Bulgaria }\end{array}$ & $\begin{array}{l}42^{\circ} 44^{\prime} 48^{\prime \prime N} \\
24^{\circ} 16^{\prime} 51^{\prime \prime E}\end{array}$ & $1630 \mathrm{~m}$ & $\begin{array}{l}\text { Bythinella hansboetersi Glöer et } \\
\text { Pešić, } 2006\end{array}$ & & 1 \\
\hline 12 & $\begin{array}{l}\text { Anton town, below the top of } \\
\text { Bolovan Hill, Bulgaria }\end{array}$ & $\begin{array}{l}42^{\circ} 46^{\prime} 16^{\prime \prime} \mathrm{N} \\
24^{\circ} 16^{\prime} 11 " \mathrm{E}\end{array}$ & $1850 \mathrm{~m}$ & Bythinella hansboetersi & & 1 \\
\hline 13 & $\begin{array}{l}\text { Sredna Gora Mts., W of Djulevo vil- } \\
\text { lage, } S \text { of Streltcha town, Bulgaria }\end{array}$ & $\begin{array}{l}42^{\circ} 27^{\prime} 16^{\prime \prime} \mathrm{N} \\
24^{\circ} 20^{\prime} 27^{\prime \prime} \mathrm{E}\end{array}$ & $393 \mathrm{~m}$ & $\begin{array}{l}\text { Bythinella srednogorica Glöer et } \\
\text { Georgiev, } 2009\end{array}$ & paratype & $3 *$ \\
\hline 14 & $\begin{array}{l}\text { Stara Planina, spring of Cherni } \\
\text { Osam, Bulgaria }\end{array}$ & $\begin{array}{l}42^{\circ} 43^{\prime} 21 " \mathrm{~N} \\
24^{\circ} 46^{\prime} 47^{\prime \prime} \mathrm{E}\end{array}$ & $2150 \mathrm{~m}$ & Bythinella hansboetersi & paratype & $1 *$ \\
\hline 15 & $\begin{array}{l}\text { West Rhodopes Mts, S of Lilkovo vil- } \\
\text { lage, near Modar peak, Bulgaria }\end{array}$ & $\begin{array}{l}41^{\circ} 52^{\prime} 39^{\prime \prime} \mathrm{N} \\
24^{\circ} 33^{\prime} 21^{\prime \prime} \mathrm{E}\end{array}$ & $1516 \mathrm{~m}$ & $\begin{array}{l}\text { Bythinella rhodopensis Glöer et } \\
\text { Georgiev, } 2009\end{array}$ & paratype & $3^{*}$ \\
\hline 16 & Mugla village, Bulgaria & $\begin{array}{l}41^{\circ} 37^{\prime} 43 " \mathrm{~N} \\
24^{\circ} 31^{\prime} 08^{\prime \prime} \mathrm{E}\end{array}$ & $1730 \mathrm{~m}$ & Bythinella hansboetersi & & 1 \\
\hline 17 & $\begin{array}{l}\text { Belasits Mts., S of Belasitsa village, } \\
\text { Bulgaria }\end{array}$ & $\begin{array}{l}41^{\circ} 21^{\prime} 07^{\prime \prime} \mathrm{N} \\
23^{\circ} 09^{\prime} 19^{\prime \prime} \mathrm{E}\end{array}$ & $687 \mathrm{~m}$ & $\begin{array}{l}\text { Bythinella slaveyae Glöer et } \\
\text { Georgiev, } 2011\end{array}$ & paratype & $3 *$ \\
\hline 18 & $\begin{array}{l}\text { Smoljan town, below Smoljanske } \\
\text { Lake, Bulgaria }\end{array}$ & $\begin{array}{l}41^{\circ} 37^{\prime} 01^{\prime \prime} \mathrm{N} \\
24^{\circ} 40^{\prime} 31^{\prime \prime} \mathrm{E}\end{array}$ & $1490 \mathrm{~m}$ & Bythinella hansboetersi & & 1 \\
\hline 19 & Smoljan town, near Amzovo, Bulgaria & $\begin{array}{l}41^{\circ} 33^{\prime} 42^{\prime \prime} \mathrm{N} \\
24^{\circ} 41^{\prime} 41^{\prime \prime} \mathrm{E}\end{array}$ & $1520 \mathrm{~m}$ & Bythinella hansboetersi & & 1 \\
\hline 20 & $\begin{array}{l}\text { Anthousa, Pindos Mts., Epirus, } \\
\text { Greece }\end{array}$ & $\begin{array}{l}39^{\circ} 39^{\prime} 29^{\prime \prime} \mathrm{N} \\
21^{\circ} 12^{\prime} 30^{\prime \prime} \mathrm{E}\end{array}$ & $967 \mathrm{~m}$ & Bythinella sp. G1 & & 1 \\
\hline 21 & Sirakon, Pindos Mts., Epirus, Greece & $\begin{array}{l}39^{\circ} 35^{\prime} 41 " \mathrm{~N} \\
21^{\circ} 06^{\prime} 16^{\prime \prime} \mathrm{E}\end{array}$ & $1032 \mathrm{~m}$ & Bythinella sp. G2 & & 1 \\
\hline 22 & $\begin{array}{l}\text { Palaiokhorion, W of Kardhitsa, } \\
\text { Thessalia, Greece }\end{array}$ & $\begin{array}{l}39^{\circ} 22^{\prime} 07^{\prime \prime} \mathrm{N} \\
21^{\circ} 37^{\prime} 53^{\prime \prime} \mathrm{E}\end{array}$ & $774 \mathrm{~m}$ & Bythinella sp. G3 & & 1 \\
\hline 23 & $\begin{array}{l}\text { between Kalivia Filaktis and Portitsa, } \\
\text { SW of Kardhitsa, Thessalia, Greece }\end{array}$ & $\begin{array}{l}39^{\circ} 19^{\prime} 35^{\prime \prime} \mathrm{N} \\
21^{\circ} 45^{\prime} 51^{\prime \prime} \mathrm{E}\end{array}$ & $681 \mathrm{~m}$ & Bythinella sp. G4 & & 1 \\
\hline 24 & $\begin{array}{l}\text { Pilion Mt., N of Dhrakia, E of Volos, } \\
\text { Greece Pilion Mt., N of Dhrakia, E of } \\
\text { Volos, Greece }\end{array}$ & $\begin{array}{l}39^{\circ} 23^{\prime} 36^{\prime \prime} \mathrm{N} \\
23^{\circ} 02^{\prime} 33^{\prime \prime} \mathrm{E} \\
39^{\circ} 24^{\prime} 35^{\prime \prime} \mathrm{N} \\
23^{\circ} 04^{\prime} 54^{\prime \prime} \mathrm{E}\end{array}$ & $\begin{array}{l}1082 \mathrm{~m} \\
860 \mathrm{~m}\end{array}$ & $\begin{array}{l}\text { Bythinella sp. G5 } \\
\text { Bythinella sp. G6 }\end{array}$ & $\begin{array}{l}(4 \mathrm{M} 24) \\
(4 \mathrm{M} 22)\end{array}$ & 1 \\
\hline 25 & $\begin{array}{l}\text { Kerasias Spring, Sivros, Lefkas Island, } \\
\text { Greece }\end{array}$ & $\begin{array}{l}38^{\circ} 40^{\prime} 15^{\prime \prime} \mathrm{N} \\
20^{\circ} 39^{\prime} 01 " \mathrm{E}\end{array}$ & $260 \mathrm{~m}$ & Bythinella sp. G7 & & 1 \\
\hline 26 & $\begin{array}{l}\text { Agtrini, S of Eptalofos, Parrnassos } \\
\text { Mts., Greece }\end{array}$ & $\begin{array}{l}38^{\circ} 34^{\prime} 58^{\prime \prime} \mathrm{N} \\
22^{\circ} 29^{\prime} 25^{\prime \prime} \mathrm{E}\end{array}$ & $1017 \mathrm{~m}$ & $\begin{array}{l}\text { Bythinella charpentieri (Roth, } \\
1855 \text { ) }\end{array}$ & & 1 \\
\hline 27 & $\begin{array}{l}\text { Kessariani Monastery, Athens, Attica, } \\
\text { Greece }\end{array}$ & $\begin{array}{l}37^{\circ} 57^{\prime} 39^{\prime \prime} \mathrm{N} \\
23^{\circ} 47^{\prime} 55^{\prime \prime} \mathrm{E}\end{array}$ & $358 \mathrm{~m}$ & Bythinella charpentieri & & 1 \\
\hline 28 & $\begin{array}{l}\text { between Bouzion and Kalianci, N } \\
\text { Peloponnese, Greece }\end{array}$ & $\begin{array}{l}37^{\circ} 53^{\prime} 39^{\prime \prime} \mathrm{N} \\
22^{\circ} 28^{\prime} 27^{\prime \prime} \mathrm{E}\end{array}$ & $841 \mathrm{~m}$ & Bythinella sp. G8 & & 1 \\
\hline 29 & $\begin{array}{l}\text { Agio Theodori, near Belitseka and } \\
\text { Koutiveika, Taigetos Mts., Greece }\end{array}$ & $\begin{array}{l}36^{\circ} 56^{\prime} 56^{\prime \prime} \mathrm{N} \\
22^{\circ} 10^{\prime} 01 " \mathrm{E}\end{array}$ & $238 \mathrm{~m}$ & Bythinella sp. G9 & & 1 \\
\hline 30 & $\begin{array}{l}\text { NNW of Ghorani. Taigetos Mts., } \\
\text { Peloponnese, Greece }\end{array}$ & $\begin{array}{l}36^{\circ} 56^{\prime} 45^{\prime \prime} \mathrm{N} \\
22^{\circ} 24^{\prime} 34^{\prime \prime} \mathrm{E}\end{array}$ & $571 \mathrm{~m}$ & Bythinella sp. G8 & & 1 \\
\hline 31 & $\begin{array}{l}\text { spring at Karavas, } \mathrm{N} \text { of Kithira Island } \\
\text { Greece }\end{array}$ & $\begin{array}{l}36^{\circ} 20^{\prime} 50^{\prime \prime} \mathrm{N} \\
22^{\circ} 56^{\prime} 58^{\prime \prime} \mathrm{E}\end{array}$ & $66 \mathrm{~m}$ & Bythinella sp. G8 & & $3^{*}$ \\
\hline \# & $\begin{array}{l}\text { spring at Żelazno, S of Kłodzko, } \\
\text { Sudety Mts., Poland }\end{array}$ & $\begin{array}{l}50^{\circ} 22^{\prime} 55^{\prime \prime} \mathrm{N} \\
16^{\circ} 40^{\prime} 02^{\prime \prime} \mathrm{E}\end{array}$ & $306 \mathrm{~m}$ & $\begin{array}{l}\text { Bythinella austriaca ehrmanni } \\
\text { Pax, } 1938\end{array}$ & topotype & $4^{*}$ \\
\hline \# & Rawka Mt., Bieszczady Mts., Poland & $\begin{array}{l}49^{\circ} 07^{\prime} 18^{\prime \prime} \mathrm{N} \\
22^{\circ} 35^{\prime} 16^{\prime \prime} \mathrm{E}\end{array}$ & $846 \mathrm{~m}$ & $\begin{array}{l}\text { Bythinella micherdzinskii } \\
\text { Falniowski, } 1980\end{array}$ & topotype & $1^{*}$ \\
\hline
\end{tabular}


mated using the bootstrap approach (FELSENSTEIN 1985). Bootstrap values for ML trees were calculated using 1,000 bootstrap replicates, the "fast" heuristic search algorithm, and the same model parameters as used for each ML analysis.

Minimum evolution (ME) and maximum parsimony (MP) were run on PAUP*; nodal support was estimated using the bootstrap approach (full heuristic search) with 1,000 replicates. PAUP was used to test the molecular clock hypothesis for COI with a likelihood ratio test (LRT) upon the estimated tree and best-fit model (FELSENSTEIN 1981, NEI \& KUMAR 2000, POSADA 2003).

In the phylogeny reconstruction for COI, five central European Bythinella species (B. austriaca, B. compressa, B. pannonica, B. robiciana and B. schmidti: Table 2), and B. micherdzinskii (Table 1) were used as outgroups. Next, the partition homogeneity test (FARRIS et al. 1995) was performed (1,000 replicates) with PAUP*, to check whether the two genes could be analysed together. The maximum likelihood heuristic search was then run for the combined molecular data. K2P (KIMURA 1980) distances for the COI data were calculated using PAUP*4.0b10 (SWOFFORD 2002). There is a rich literature concerning the use of K2P distances for COI data, thus we chose this distance for the sake of comparison of the levels of differentiation.

Table 2. GenBank Accession Numbers and references of COI sequences already published

\begin{tabular}{|c|c|c|c|}
\hline \multicolumn{2}{|l|}{ Species } & GenBankAN & References \\
\hline \multicolumn{2}{|c|}{ Bythinella austriaca (Frauenfeld, 1857) } & FJ545132 & FALNIOWSKI et al. (2009a) \\
\hline \multicolumn{2}{|c|}{ B. compressa (Frauenfeld, 1857) } & AF367653 & SZAROWSKA \& WILKE (2004) \\
\hline \multicolumn{2}{|c|}{ B. pannonica (Frauenfeld, 1865) } & AY222660 & SZAROWSKA \& WILKE (2004) \\
\hline \multicolumn{2}{|l|}{ B. robiciana (Clessin, 1890) } & AY273998 & SZAROWSKA \& WILKE (2004) \\
\hline \multicolumn{2}{|l|}{ B. schmidti (Küster, 1852) } & AY222649 & SZAROWSKA \& WILKE (2004) \\
\hline \multicolumn{2}{|l|}{ B. dacica Grossu, 1946} & FJ545011 & FALNIOWSKI et al. (2009a) \\
\hline \multicolumn{2}{|c|}{ B. calimanica Falniowski, Szarowska et Sirbu, 2009} & FJ545084 & FALNIOWSKI et al. (2009a) \\
\hline \multicolumn{2}{|l|}{ B. molcsanyi $\mathrm{H}$. Wagner, 1941} & FJ545062 & FALNIOWSKI et al. (2009a) \\
\hline \multicolumn{2}{|c|}{ B. grossui Falniowski, Szarowska et Sirbu, 2009} & FJ545106 & FALNIOWSKI et al. (2009a) \\
\hline \multicolumn{2}{|c|}{ B. radomani Falniowski, Szarowska et Sirbu, 2009} & FJ545069 & FALNIOWSKI et al. (2009a) \\
\hline \multicolumn{2}{|c|}{ B. viseuiana Falniowski, Szarowska et Sirbu, 2009} & FJ545097 & FALNIOWSKI et al. (2009a) \\
\hline \multicolumn{2}{|c|}{ B. hansboetersi Glöer et Pešić, 2006, locality 11} & GQ152534 & FALNIOWSKI et al. (2009b) \\
\hline & locality 12 & GQ152530 & FALNIOWSKI et al. (2009b) \\
\hline & locality 16 & GQ152538 & FALNIOWSKI et al. (2009b) \\
\hline & locality 18 & GQ152518 & FALNIOWSKI et al. (2009b) \\
\hline & locality 19 & GQ152523 & FALNIOWSKI et al. (2009b) \\
\hline \multirow[t]{2}{*}{ B. charpentieri (Roth, 1855) } & locality 26 & JF314213 & FALNIOWSKI \& SZAROWSKA (2011) \\
\hline & locality 27 & JF314160 & FALNIOWSKI \& SZAROWSKA (2011) \\
\hline Bythinella sp. G1 & locality 20 & JF314305 & FALNIOWSKI \& SZAROWSKA (2011) \\
\hline Bythinella sp. G2 & locality 21 & JF314298 & FALNIOWSKI \& SZAROWSKA (2011) \\
\hline Bythinella sp. G3 & locality 22 & JF314285 & FALNIOWSKI \& SZAROWSKA (2011) \\
\hline Bythinella sp. G4 & locality 23 & JF314284 & FALNIOWSKI \& SZAROWSKA (2011) \\
\hline Bythinella sp. G5 & locality 24 & JF314259 & FALNIOWSKI \& SZAROWSKA (2011) \\
\hline Bythinella sp. G6 & locality 24 & JF314272 & FALNIOWSKI \& SZAROWSKA (2011) \\
\hline Bythinella sp. G7 & locality 25 & JF314242 & FALNIOWSKI \& SZAROWSKA (2011) \\
\hline \multirow[t]{2}{*}{ Bythinella sp. G8 } & locality 28 & JF314141 & FALNIOWSKI \& SZAROWSKA (2011) \\
\hline & locality 30 & JF314074 & FALNIOWSKI \& SZAROWSKA (2011) \\
\hline Bythinella sp. G9 & locality 29 & JF314083 & FALNIOWSKI \& SZAROWSKA (2011) \\
\hline \multicolumn{2}{|c|}{ Bithynia tentaculata (Linnaeus, 1758) } & AF367643 & WILKE et al. (2001) \\
\hline \multicolumn{2}{|c|}{ Marstoniopsis insubrica (Küster, 1853) } & AY027813 & FALNIOWSKI \& WILKE (2001) \\
\hline
\end{tabular}




\section{RESULTS}

In total, we analysed 63 sequences of COI, $570 \mathrm{bp}$ long. Two of the sequences represented the outgroup species (Bithynia tentaculata and Marstoniopsis insubrica: Table 2); six came from Romania (FALNIOWSKI et al. 2009b); 12 from Greece (FALNIOWSKI \& SZAROWSKA 2011); five from Bulgaria (FALNIOWSKI et al. 2009a); 33 sequences were new (GenBank Accession Numbers: JQ639854-JQ639886). All the 61 sequences of 18S, 450 bp long, were new (GenBank Accession Numbers: JQ639793-JQ639853).
Saturation test of XIA et al. (2003) showed little saturation in our COI data: $I_{s s}=0.309, I_{s s . c}=0.707$ for symmetrical topology, d.f. $=190, \mathrm{p}=0.000$, and $I_{s s . c}=0.385$ for asymmetrical topology, d.f. $=190, p=0.0079$.

For the COI the Akaike Information Criterion (AIC) with ModelTest selected the model $G T R+I+\Gamma$, with base frequencies: $\mathrm{A}=0.3409, \mathrm{C}=0.2163, \mathrm{G}=$ $0.1225, \mathrm{~T}=0.3204$; substitution rate matrix: $[\mathrm{A}-\mathrm{C}]=$ 272.8169, $[\mathrm{A}-\mathrm{G}]=4222.1479,[\mathrm{~A}-\mathrm{T}]=206.2349,[\mathrm{C}-\mathrm{G}]$ $=438.3951,[\mathrm{C}-\mathrm{T}]=2715.1609,[\mathrm{G}-\mathrm{T}]=1.0000$, pro-

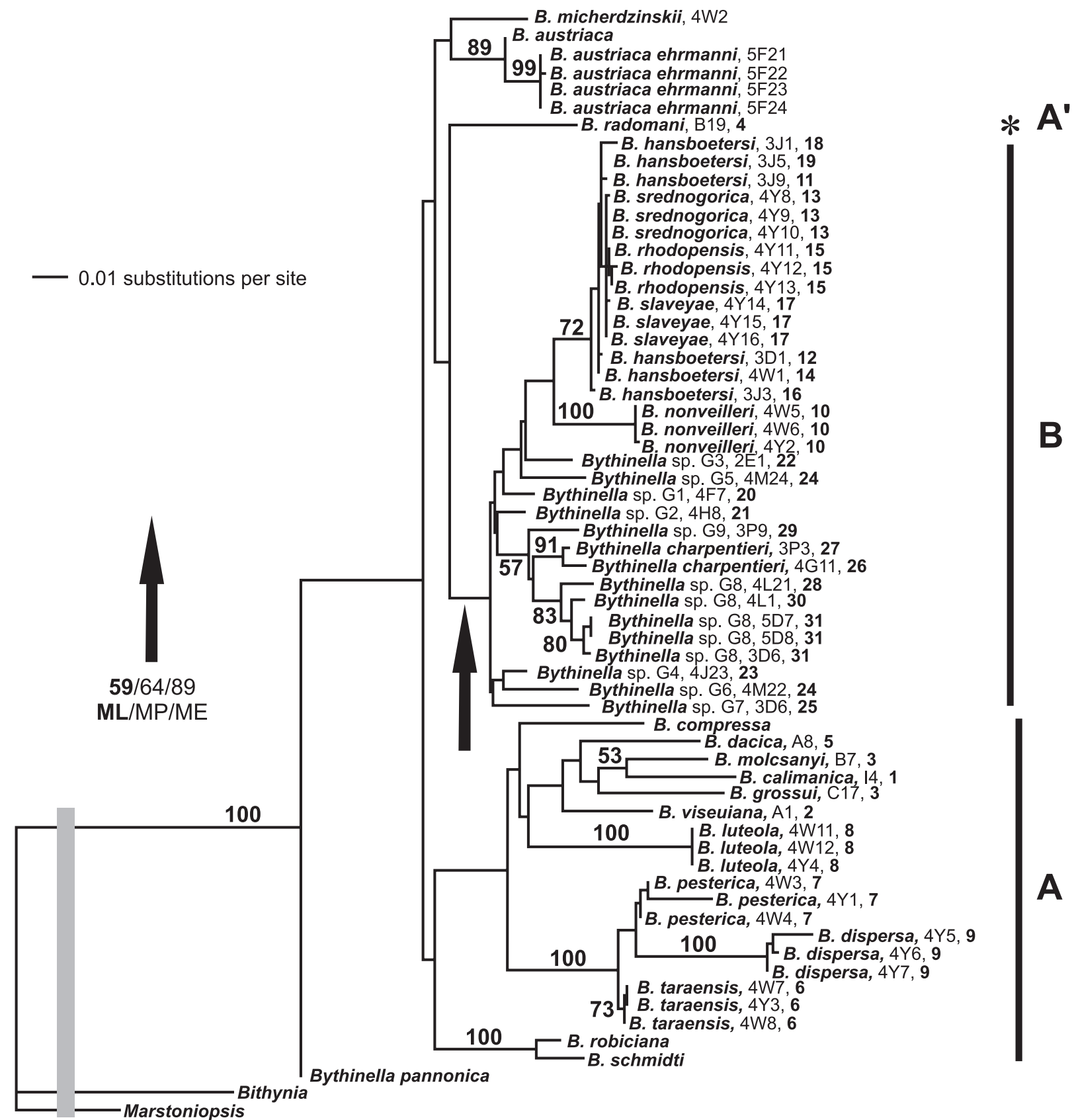

Fig. 2. Maximum likelihood phylogram of the studied Bythinella. Bootstrap supports: ML (in bold)/MP/ME/NJ are given for each clade; locality number in bold for each terminal branch. Arrow indicates the bifurcation between the two main Balkan clades (A and B) of Bythinella, whose border is marked with dotted line in Fig. 1. Shaded rectangle indicates proportional shortening of the branches connecting the ingroup with the outgroup taxa 
portion of invariable sites: $(\mathrm{I})=0.6519$, and $\Gamma$ distribution with the shape parameter $=2.5072$. The Likelihood Ratio Test (LRT) for the COI resulted in insignificant values $(p=0.023)$, so we rejected the molecular clock hypothesis.

The partition homogeneity test $(\mathrm{p}=0.732)$ showed that the two genes could be analysed together. For the combined data set the Akaike Information Criterion (AIC) with ModelTest selected the model $\mathrm{HKY}+\mathrm{I}+\Gamma$, with base frequencies: $\mathrm{A}=0.3077, \mathrm{C}=0.2397, \mathrm{G}=$ $0.1821, \mathrm{~T}=0.2704$; substitution rate matrix: $\mathrm{ti} / \mathrm{tv}$ ratio: 5.2693 , proportion of invariable sites: $(\mathrm{I})=0.7340$, and $\Gamma$ distribution with the shape parameter $=1.0465$.

Figure 2 shows the ML tree computed for the combined data, in which Bythinella pannonica from Slovakia forms a distinct Bythinella clade closest to the root. $B$. micherdzinski, B. austriaca and B. austriaca ehrmanni from Poland form another clade. The Balkan taxa form two clades that are distinct but weakly supported (59/64/89, depending on the technique): clade A (Fig. 2) consisting of populations 1-9 (Table 1), $B$. robiciana, and B. schmidti, although population 4 , representing B. radomani (marked with asterisk in Fig. 2) falls between the clade of the Polish Bythinella and the other Balkan clade (Fig. 2: A'). The second large Balkan clade (B in Fig. 2) clusters all the other Balkan populations 10-31 (Table 1) (this bifurcation is marked with arrow in Fig. 2, the dotted line in Fig. 1 indicates the geographic border between the two large clades). Within clade A the differences between the nominal taxa, reflected in branch lengths (Fig. 2), as well as in K2P distances (within group mean: 0.1004), are greater than in clade $\mathrm{B}$ (within group mean: 0.0499). The pairwise K2P distances between the two clades are 0.09-0.1744, mean 0.1224 , net mean 0.0473 .

\section{DISCUSSION}

FALNIOWSKI \& SZAROWSKA (2011) demonstrated that in the Greek Bythinella there was much less interspecific differentiation than in the Romanian Bythinella, and the diversity decreased from north to south. In this study we observed the same general pattern throughout the East Balkans. The somewhat larger distances between the studied Romanian populations may contribute to this pattern. However, this cannot be the main explanation, as between the two sympatric species at locality 4 in Romania the distance was not smaller than between the allopatric Romanian species. Thus another explanation should be invoked.

The Romanian Pleistocene refugium, or rather a set of small territories with a mild climate, was situated farther to the north than the other European refugia. In fact, it was rather a group of nunatak-like habitats, the fauna of which was probably under a strong influence of unstable climatic conditions. Across the country there were several scattered glacial refugia, which, during the interglacials and especially in the postglacial, served as distribution centres (FALNIOWSKI et al. 2009b). The expansions and contractions of the glacier proceeded by way of climatic changes and were followed by the contractions and expansions of the nunatak refugia, thus Bythinella ranges in the studied territory may have promoted genetic differentiation within the genus (HEWITT 1989). This contrasts with the south Balkan (Greek) refugium, which was rather continuous geographically and characterised by relatively stable conditions. Perhaps those more stable conditions during the Pleistocene are reflected in smaller differences between the species of Bythinella in the south of the Balkans.

The two large clades in the inferred phylogeny are well-defined geographically. The dotted line in the map (Fig. 1) marks the border between these two clades. The first (clade A, localities 1-9) includes the six Romanian species (localities 1-5), the three Montenegro populations (localities 6 and 8-9), and one of two Serbian populations of Bythinella (locality 7). This group is closer to B. pannonica from Slovakia and Hungary, B. austriaca and B. micherdzinskii from Poland, $B$. compressa from Germany, as well as $B$. schmidti and $B$. robiciana from Slovenia, and to the outgroup. In fact, $B$. radomani from locality 4 (A' in Fig. 2) clusters between the Polish taxa and the second large clade. This may be explained, in part, by the location of that species in the northernmost part of Romania, thus not far from the Polish localities. One can speculate that B. radomani invaded the locality from the north/northwest.

The second clade (clade B) clusters all populations from Greece and Bulgaria together with one population (10) from Serbia. As stated above, the genetic diversity, as marked in COI, was lower within this clade. It is noteworthy that presumably only one species occurs across Bulgaria. As could be seen in the map (Fig. 1), there are, unfortunately, no samples close to the border marked on the map, thus its localisation is a very rough estimate only. Moreover, we cannot prove the real distinctness of those two clades, since their bootstrap supports are rather low (Fig. 2). Thus we can only hypothesise about this part of the history of Bythinella in the Balkans.

There are no data on the fossil occurrence of Bythinella in the studied part of Europe. Fossil Bythinella were found in the Italian Pliocene/Pleistocene boundary (southern margin of the Alps, north of Bergamo: ESU \& GIANOLLA 2009). In the Balkans - their southern part, at least - the history of this genus cannot be shorter. As we rejected the molecular clock hypothesis 
- and had not any precise point of callibration that would enable us to apply the techniques of relaxing molecular clock assumptions - we could not put a precise time frame on our tree. However, we can use, with some caution, the estimate of FALNIOWSKI \& SZAROWSKA (2011), dating the origin of the SE clade (containing all the Greek and Bulgarian populations), thus the time of cladogenesis marked with the arrow in Fig. 2 certainly was before $4.341 \pm 0753$ MYA (middle Pliocene). This coincides in time with the existence of the Dacic Basin, a vast water body that separated the present Carpathians from the present middle Bulgaria (POPOV et al. 2004, 2006, POPESCU et al. 2009). The Dacic Basin, part of the Paratethys, connected with the Pannonian Basin in the west, the Euxinian Basin in the east, and directly with the present Aegean Sea in the south, eventually decreasing in size, was still present till the latest Pliocene, 1.8 MYA. The Dacic Basin most probably separated the ancestors of the two large clades. Later, in the Pleistocene, the unstable fluvio-

\section{REFERENCES}

AKAIKE H. 1974. A new look at the statistical model identification. IEEE Transactions of Automatic Control 19: 716-723. doi: 10.1109/TAC.1974.1100705

Benke M., BRÄNDle M., Albrecht C., Wilke T. 2009. Pleistocene phylogeography and phylogenetic concordance in cold-adapted spring snails (Bythinella spp.). Molec. Ecol. 18: 890-903. doi: 10.1111/j.1365-294X.2008.04073.x

Bichain J.-M., GAUbert P., SAMADI S., Boisselier-Dubayle M.-C. 2007a. A gleam in the dark: Phylogenetic species delimitation in the confusing spring-snail genus Bythinella Moquin-Tandon, 1856 (Gastropoda: Rissooidea: Amnicolidae). Mol. Phylogenet. Evol. 45: 927-941. doi: 10.1016/j.ympev.2007.07.018

Bichain J.-M., Boisselier-DubaYle M.-C., BOUChet P., SAMADI S. 2007b. Species delimitation in the genus Bythinella (Mollusca: Caenogastropoda: Rissooidea): a first attempt combining molecular and morphometrical data. Malacologia 49: 293-311. doi: 10.4002/0076-2997-49.2.293

DAVIS G. M. 1992. Evolution of prosobranch snails transmitting Asian Schistosoma: coevolution with Schistosoma: a review. Prog. Clin. Parasitol. 3: 145-204.

ESU D., GianOlLa D. 2009. The malacological record from the Plio-Pleistocene Leffe Basin (Bergamo, Northern Italy). Quatern. Int. 204: 11-19. doi: 10.1016/j.quaint.2008.08.001

FALNIOWSKI A. 1980. Bythinella micherdzinskii n. sp. - new species from the family Bythinellidae s. Radoman (Gastropoda, Prosobranchia). Bull. Acad. Pol. Sci., Ser. sci. biol. Cl. 2, 28: 225-230.

FALNIOWSKi A. 1987. Hydrobioidea of Poland (Prosobranchia: Gastropoda). Folia Malacol. 1: 1-122.

FALNIOWSKI A. 1992. Genus Bythinella Moquin-Tandon, 1855, in Poland (Gastropoda, Prosobranchia, Hydrobiidae. In: lacustrine system in SW. Bulgaria and northern Greece, with glaciers present in the Pirin and Rila Mts (ZAGORCHEV 2007), probably formed effective, temporary barriers for Bythinella, and may have caused its extinction in the vast part of Bulgaria. Considering the data known so far, the small differences among the nine Bulgarian populations may reflect the short history of Bythinella in the area that most probably was recolonised from the south not earlier than in the late Pleistocene.

\section{ACKNOWLEDGEMENTS}

The study was supported by a grant from the Polish Ministry of Science and Higher Education (PB 2443/P01/2006/31) to ANDRZEJ FALNIOWSKI. We would like to thank the two anonymous reviewers for their valuable comments on the previous version of the manuscript.

GitTenberger E., Goud J. (eds). Proc. 9th Int. Malac. Congr. Edinburgh, 31 August-6 September 1986. Unitas Malacologica, Leiden: 135-138.

FALNIOWSKI A. 2003. Metody numeryczne w taksonomii. Wydawnictwo Uniwersytetu Jagiellońskiego, Kraków.

FALNIOWSKi A., HORSAK M., SZAROWSKA M. 2009a. Bythinella hansboetersi Glöer et Pešić, 2006 (Gastropoda: Rissooidea) in Bulgaria: its morphology, molecular distinctness, and phylogeography. Folia Malacol. 17: 11-20. doi: $10.2478 /$ v10125-009-0002-3

FALNIOWSKI A., SzAROWSKA M. 2011. Radiation and phylogeography in a spring snail Bythinella (Mollusca: Gastropoda: Rissooidea) in continental Greece. Ann. Zool. Fennici 48: 67-90.

FALNiOWSki A., SZAROWSKA M., SIRBU I. 2009b. Bythinella Moquin-Tandon, 1856 (Gastropoda: Rissooidea: Bythinellidae) in Romania: species richness in a glacial refugium. J. Nat. Hist. 43: 2955-2973.

doi: 10.1080/00222930903359636

FALNIOWSKi A., SZAROWSKA M., SiRBU I. 2009c. Bythinella Moquin-Tandon, 1856 (Gastropoda: Rissooidea: Bythinellidae) in Romania: its morphology with description of four new species. Folia Malacol. 17: 21-36. doi: 10.2478/v10125-009-0003-2

FALNIOWSKI A., Wilke T. 2001. The genus Marstoniopsis (Gastropoda: Rissooidea): intra- and intergeneric phylogenetic relationships. J. Moll. Stud. 67: 483-488. doi:10.1093/mollus/67.4.483

FARRIS J. S., KÄLlERSJÖ M., KlugE A. G., Bult C. 1995. Testing significance of incongruence. Cladistics 10: 315-319. doi: 10.1111/j.1096-0031.1994.tb00181.x

FELSENSTEIN J. 1981. Evolutionary trees from DNA sequences: a maximum-likelihood approach. J. Molec. Evol. 17: 368-376. doi: 10.1007/BF01734359 
FELSENSTEIN J. 1985. Confidence limits on phylogenies: an approach using the bootstrap. Evolution 39: 783-791. doi: $10.2307 / 2408678$

FOLMER O., BLACK M., HOEH W., LUTZ R. A., VRIJENHOEK R. C. 1994. DNA primers for amplification of mitochondrial cytochrome c oxidase subunit I from diverse metazoan invertebrates. Mol. Mar. Biol. Biotechnol. 3: 294-299.

GIUSTI F., PEZzOLI E. 1977. Primo contributo alla revisione del genere Bythinella in Italia. Natura Bresciana, Annurario del Museo Civico di Storia Naturale di Brescia 14: 3-80.

GLÖER P. 2002. Die Süsswassergastropoden Nord- und Mitteleuropas, Bestimmungsschlüssel, Lebensweise, Verbreitung. Die Tierwelt Deutschlands, 73. Teil. ConchBooks, Hackenheim.

GLÖER P. 2008. Three new hydrobioid species from Serbia (Mollusca, Gastropoda, Hydrobiidae). In: PAVIĆEvić D., M. PERREAU (eds). Advances in the studies of the subterranean and epigean fauna of the Balkan Peninsula. Volume dedicated to the memory of Guido Nonvellier. Monograph 22, Institute for Nature Conservation of Serbia, pp. 349-356.

GLÖER P., GEORGIEV D. 2009. New Rissooidea from Bulgaria (Gastropoda: Rissooidea). Mollusca 27: 123-136.

GLÖER P., PEŠIĆ V. 2006. Bythinella hansboetersi n. sp., a new species from Bulgaria. Heldia 6: 11-15.

GLÖER P., PEŠIĆ V. 2010. The freshwater snails of the genus Bythinella Moquin-Tandon (Gastropoda: Rissooidea: Hydrobiidae) from Montenegro. Arch. Biol. Sci., Belgrade 62: 441-447.

HAASE M., Wilke T., MiLdNER P. 2007. Identifying species of Bythinella (Caenogastropoda: Rissooidea): A plea for an integrative approach. Zootaxa 1563: 1-16.

HALL T. A. 1999. BioEdit: a user-friendly biological sequence alignment editor and analysis program for Windows 95/98/NT. Nucleic Acids Symp. Ser. 41: 95-98.

HewitT G. M. 1989. The subdivision of species by hybrid zones. In: OTTE D., ENDLERJ. A. (eds). Speciation and its consequences. Sinauer Associates Inc. Publishers, Sunderland, Massachusetts, pp. 85-110.

HewitT G. M. 1996. Some genetic consequences of ice ages, and their role in divergence and speciation. Biol. J. Linn. Soc. 58: 247-276.

HewITT G. M. 2000. The genetic legacy of the Quaternary ice ages. Nature 405: 907-913. doi: 10.1038/35016000

HiLlis D. M., MABLE B. K., LARSON A., DAVIS S. K., ZiMMER E. A. 1996. Nucleic acids IV: Sequencing and cloning. In: Hillis D. M., MORITZ L., Mable B. K. (eds). Molecular Systematics. Second Edition. Sinauer Associates Inc. Publishers, Sunderland, Massachusetts, pp. 321-381.

JungBluth J. H., Boeters H. D. 1977. Zur Artabgrenzung bei Bythinella dunkeri und bavarica (Prosobranchia). Malacologia 16: 143-147.

KIMURA M. 1980. A simple method for estimating evolutionary rate of base substitutions through comparative studies of nucleotide sequences. J. Mol. Evol. 16: 111-120. doi: 10.1007/BF01731581

MADDISON D. R., MADDISON W. P. 2002. MACCLADE. Ver. 4.05. [Computer software and manual]. Sinauer Associates Inc. Publishers, Sunderland, Massachusetts.
NEI M., KumaR S. 2000. Molecular evolution and phylogenetics. Oxford University Press, Oxford.

PAlumbi S. R. 1996. Nucleic Acids II: The Polymerase Chain Reaction. In: Hillis D. M, MORITZ C., MABle B. K. (eds). Molecular Systematics. Second Edition. Sinauer Associates Inc., Sunderland, Massachusetts, pp. 205-247.

Petit R. J., Aguinagalde I., DE Beaulieu J.-L., BitTKaU C., BREWER S., CHEDDADI R., ENNOS R., FINESCHI S., GRIVET D., Lascoux M., MOHANTY A., MÜlleR-STARCK G., DEMesure-Musch B., Palmé A., Martín J. P., REndell S., VENDRAMIN G. 2003. Glacial refugia: hotspots but not melting pots of genetic diversity. Science 300: 1563-1565. doi: 10.1126/science. 1083264

Popescu S.-M., Dalesme F., Jouannic G., Escarguel G., HeAd M., Melinte-Dobrinescu M.-C., SÜtO-Szentai M., Bakrac K., Clauzon G., SuC J. P. 2009. Galeacysta etrusca complex: dinoflagellate cyst marker of Paratethyan influxes to the Mediterranean Sea before and after the peak of the Messinian Salinity Crisis. Palynology 33: 105-134.

Popov S. V., Rögl F., Rozanov A. Y., Steininger F. F., SHCHERBA I. G., KOVAC M. 2004. Lithological-paleogeographic maps of the Paratethys. 10 maps Late Eocene to Pliocene. Courier Forschungsinstitut Senckenberg 250: $1-46$.

Popov S. V., ShChERba I. G., Ilyina L. B., NeVESSKAYA L. A., PARAMONOVA N. P., KHONDKARIAN S. O., MAGYAR I. 2006. Late Miocene to Pliocene palaeogeography of the Paratethys and its relation to the Mediterranean. Palaeogeogr. Palaeoclimatol. Palaeoecol. 238: 91-106. doi: $10.1016 /$ j.palaeo.2006.03.020

POSADA D. 2003. Selecting models of evolution. In: SALEMI M., VANDAMME A.- M. (eds). The phylogenetic handbook. A practical approach to DNA and protein phylogeny. Cambridge University Press, Cambridge, pp. 256-282.

POSADA D., BUCKLEY T. R. 2004. Model selection and model averaging in phylogenetics: Advantages of Akaike Information Criterion and Bayesian approaches over likelihood ratio tests. Syst. Biol. 53: 793-808. doi: 10.1080/10635150490522304

Posada D., Crandall K. A. 1998. Modeltest: testing the model of DNA substitution. Bioinformatics 14: 817-818. doi: 10.1093/bioinformatics/14.9.817

RADOMAN P. 1976. Speciation within the family Bythinellidae on the Balkans and Asia Minor. Z. Zool. System. Evol. 14: 130-152. doi: 10.1111/j.1439-0469.1976.tb00522.x

RADOMAN P. 1983. Hydrobioidea a superfamily of Prosobranchia (Gastropoda). I; Systematics. Monographs, Department of Sciences, Serbian Academy of Sciences and Arts 547(57): 1-256.

RADOMAN P. 1985. Hydrobioidea, a superfamily of prosobranchia (Gastropoda). II: Origin, zoogeography, evolution in the Balkans and Asia Minor. Monographs, Faculty of Science, Department of Biology, Institute of Zoology Beograd 1: 1-173.

SCHMITT T. 2007. Molecular biogeography of Europe: Pleistocene cycles and postglacial trends. Front. Zool. 4: 11. doi: 10.1186/1742-9994-4-11

SOBER E. 2002. Instrumentalism, parsimony, and the Akaike framework. Philos. Sci. 69: 112-123. doi: 10.1086/341839 
SWOFFORD D. L. 2002. PAUP*- Phylogenetic analysis using parsimony (* and other methods). Ver. 4. [Computer software and manual]. Sinauer Associates Inc., Sunderland, Massachusetts.

SWOFFORD D. L., Olsen G. J., WADDEll P. J., Hillis D. M. 1996. Phylogenetic inference. In: HILlIS D. M., MORITZ C., MABLE B. K. (eds). Molecular Systematics Second ed. Sinauer Associates Inc., Sunderland, Massachusetts, pp. $407-514$

SZAROWSKA M., WiLKE T. 2004. Sadleriana pannonica (Frauenfeld, 1865): a lithoglyphid, hydrobiid or amnicolid taxon? J. Moll. Stud. 70: 49-57. doi: 10.1093/mollus/70.1.49

Thompson J. D., Gibson T. J., Plewniak F., JEANMOUgin F., HigGins D. G. 1997. The ClustalX windows interface: flexible strategies for multiple sequence alignment aided by quality analysis tools. Nucleic Acids Res. 24: 4876-4882. doi: 10.1093/nar/25.24.4876

Vogel J. C., Rumsey F. J., SChneller J. J., Barrett J. A., GIBBY M. 1999. Where are the glacial refugia in Europe? Evidence from pteridophytes. Biol. J. Linn. Soc. 66: 23-37.
Wilke T., DAVIS G. M., FALNIOWSKI A., GIUSTI F., BODON M., SZAROWSKA M. 2001. Molecular systematics of Hydrobiidae (Gastropoda: Rissooidea): testing monophyly and phylogenetic relationships. Proc. Acad. Nat. Sci. Philad. 151: 1-21. doi:10.1635/0097-3157(2001)151[0001:MSOHMG]2.0. $\mathrm{CO} ; 2$

XIA X. 2000. Data analysis in molecular biology and evolution. Kluwer Academic Publishers. Boston/Dordrecht/ London.

Xia X., Xie Z., SAlemi M., Chen L., WANG Y. 2003. An index of substitution saturation and its application. Mol. Phylogenet. Evol. 26: 1-7. doi: 10.1016/S1055-7903(02)00326-3

ZaGorCHEV I. 2007. Late Cenozoic development of the Strouma and Mesta fluviolacustrine systems, SW Bulgaria and northern Greece. Quaternary Sci. Rev. 26: 2783-2800. doi: 10.1016/j.quascirev.2007.07.017

Received: May 11th, 2011

Revised: August 2nd, 2011 Accepted: August 4th, 2011 